Supplement of Hydrol. Earth Syst. Sci., 19, 4055-4066, 2015

http://www.hydrol-earth-syst-sci.net/19/4055/2015/

doi:10.5194/hess-19-4055-2015-supplement

(C) Author(s) 2015. CC Attribution 3.0 License.

(c) (i)

Hydrology and Earth System

Sciences

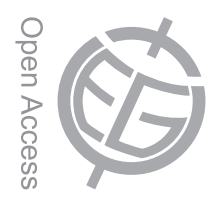

Supplement of

\title{
The effect of empirical-statistical correction of intensity-dependent model errors on the temperature climate change signal
}

\section{A. Gobiet et al.}

Correspondence to: A. Gobiet (andreas.gobiet@zamg.ac.at)

The copyright of individual parts of the supplement might differ from the CC-BY 3.0 licence. 


\section{The effect of empirical-statistical correction of intensity- \\ 2 dependent model errors on the climate change signal}

4

\section{Supplementary Figures}
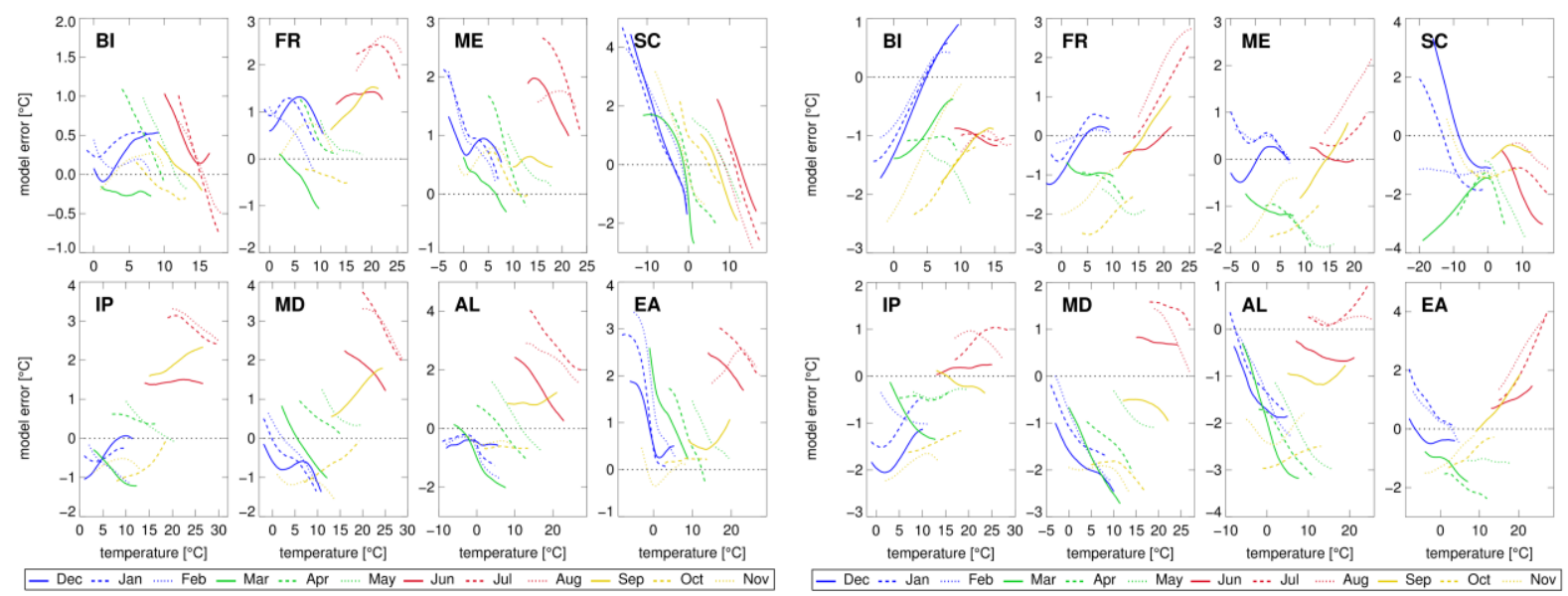

Figure S1: Temperature error characteristics (model minus observation) of the C4I RCA HadCM3Q16 (left panels) and CNRM ALADIN ARPEGE5 (right panels) RCMs in eight sub-regions of Europe (sub-panels) and each month of the year.
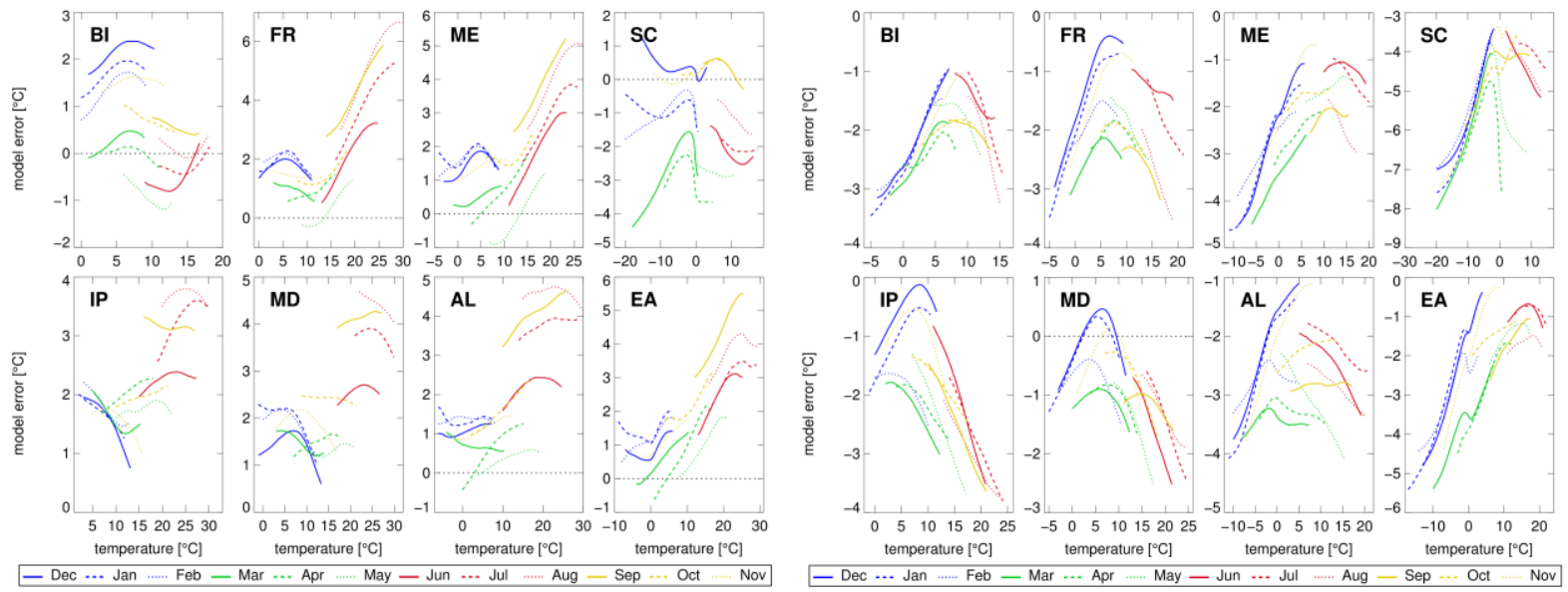

11 Figure S2 Temperature error characteristics (model minus observation) of the DMI HIRHAM 12 ARPEGE (left panels) and DMI HIRHAM BCM (right panels) RCMs in eight sub-regions of 13 Europe (sub-panels) and each month of the year. 

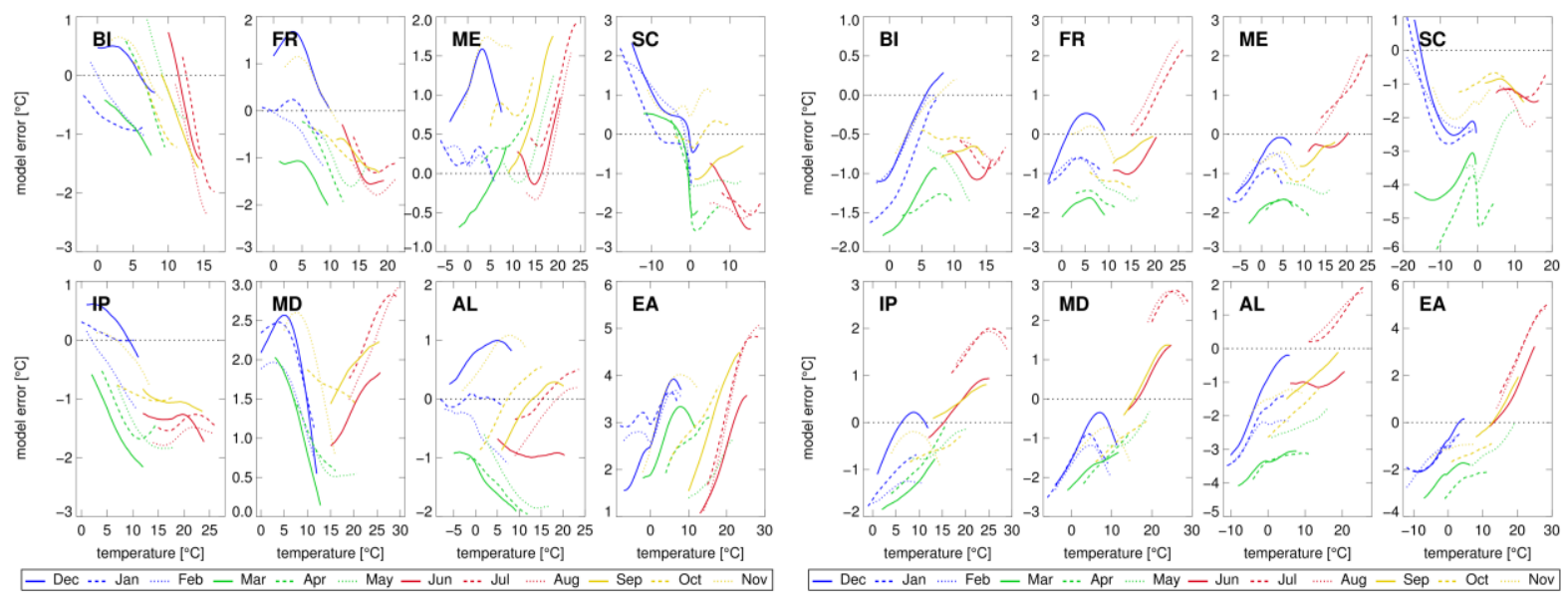

2 Figure S3: Temperature error characteristics (model minus observation) of the DMI 3 HIRHAM ECHAM5-r3 (left panels) and ETHZ CLM HadCM3Q0 (right panels) RCMs in 4 eight sub-regions of Europe (sub-panels) and each month of the year.
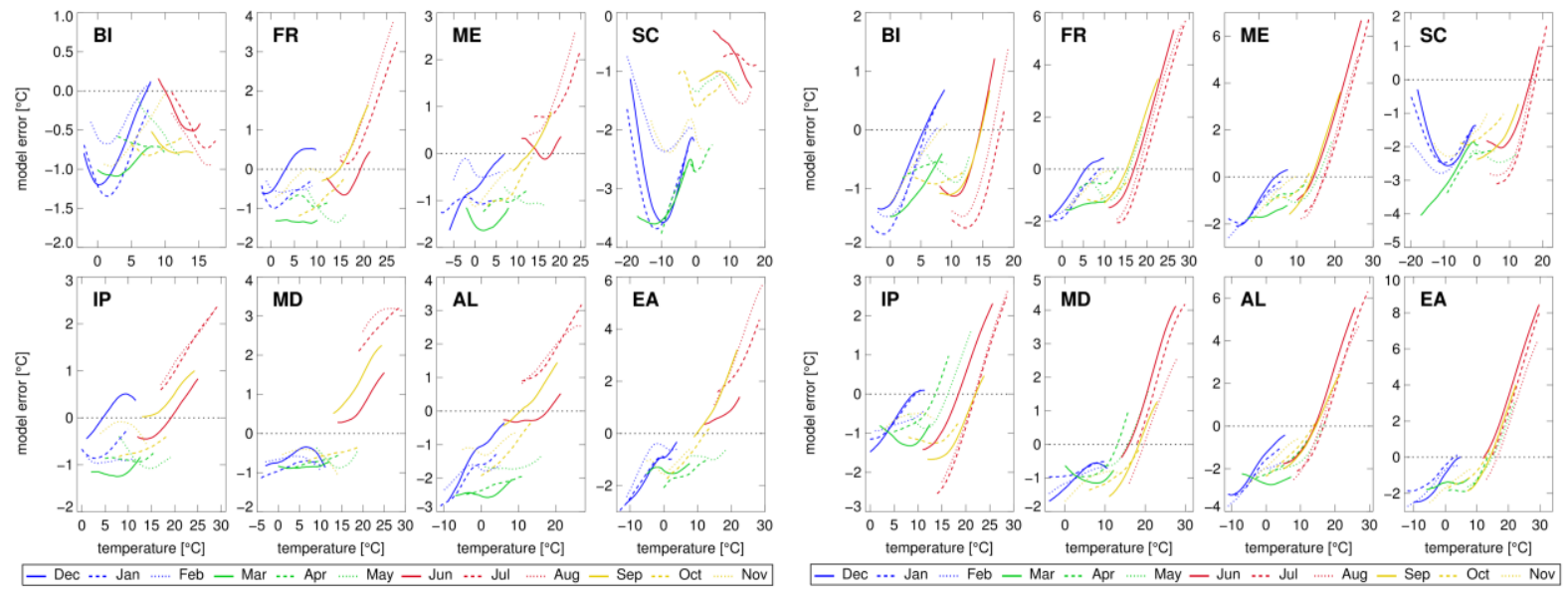

6 Figure S4: Temperature error characteristics (model minus observation) of the HC 7 HadRM3Q0 HadCM3Q0 (left panels) and HC HadRM3Q3 HadCM3Q3 (right panels) RCMs 8 in eight sub-regions of Europe (sub-panels) and each month of the year. 

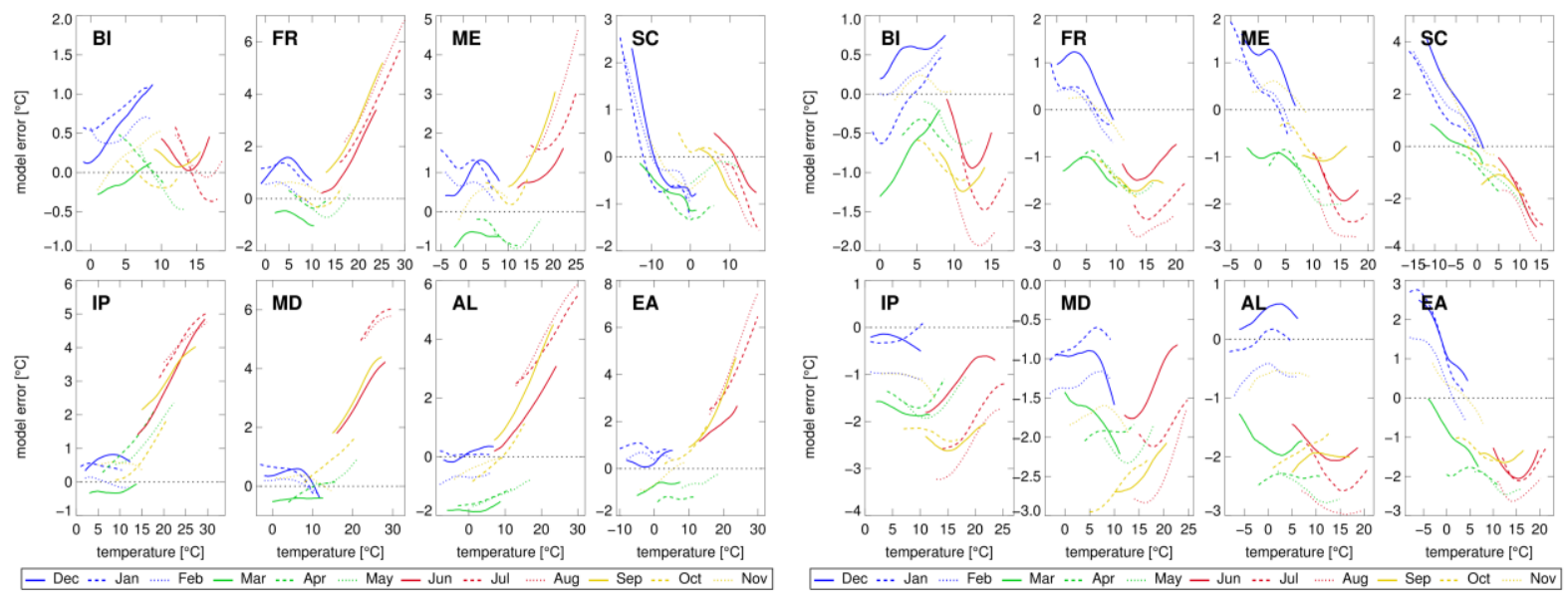

2 Figure S5: Temperature error characteristics (model minus observation) of the HC 3 HadRM3Q16 HadCM3Q16 (left panels) and ICTP RegCM ECHAM5-r3 (right panels) RCMs

4 in eight sub-regions of Europe (sub-panels) and each month of the year.
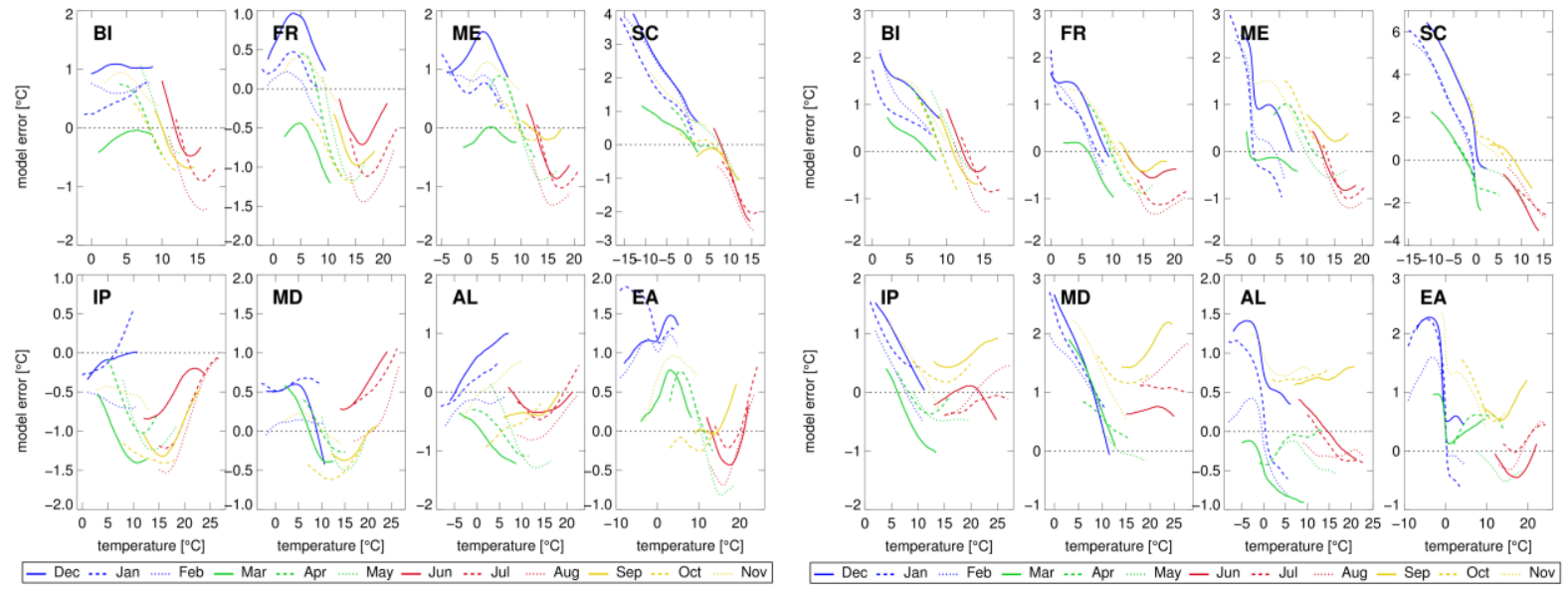

6 Figure S6: Temperature error characteristics (model minus observation) of the KNMI 7 RACMO ECHAM5-r3 (left panels) and MPI REMO ECHAM5-r3 (right panels) RCMs in 8 eight sub-regions of Europe (sub-panels) and each month of the year. 

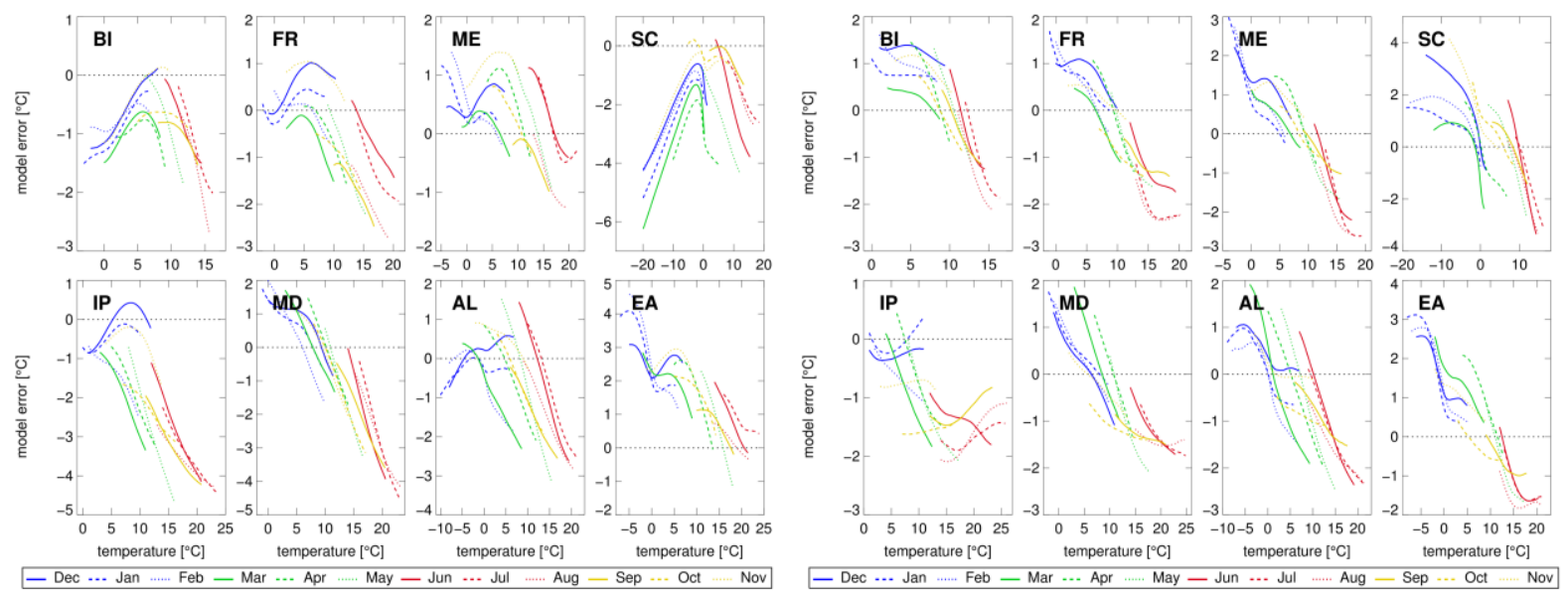

2 Figure S7: Temperature error characteristics (model minus observation) of the SMHI RCA 3 BCM (left panels) and SMHI RCA ECHAM5-r3 (right panels) RCMs in eight sub-regions of 4 Europe (sub-panels) and each month of the year.

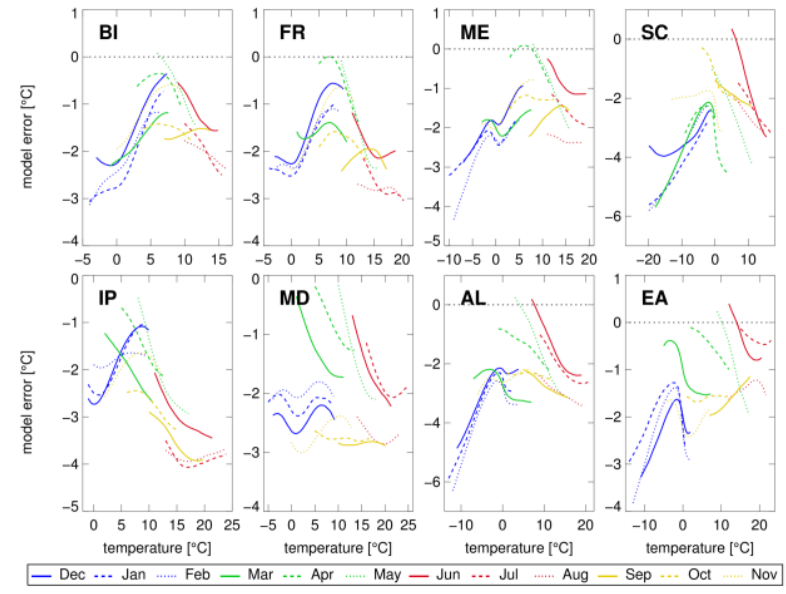

6 Figure S8: Temperature error characteristics (model minus observation) of the SMHI RCA 7 HadCM3Q3 RCM in eight sub-regions of Europe (sub-panels) and each month of the year. 

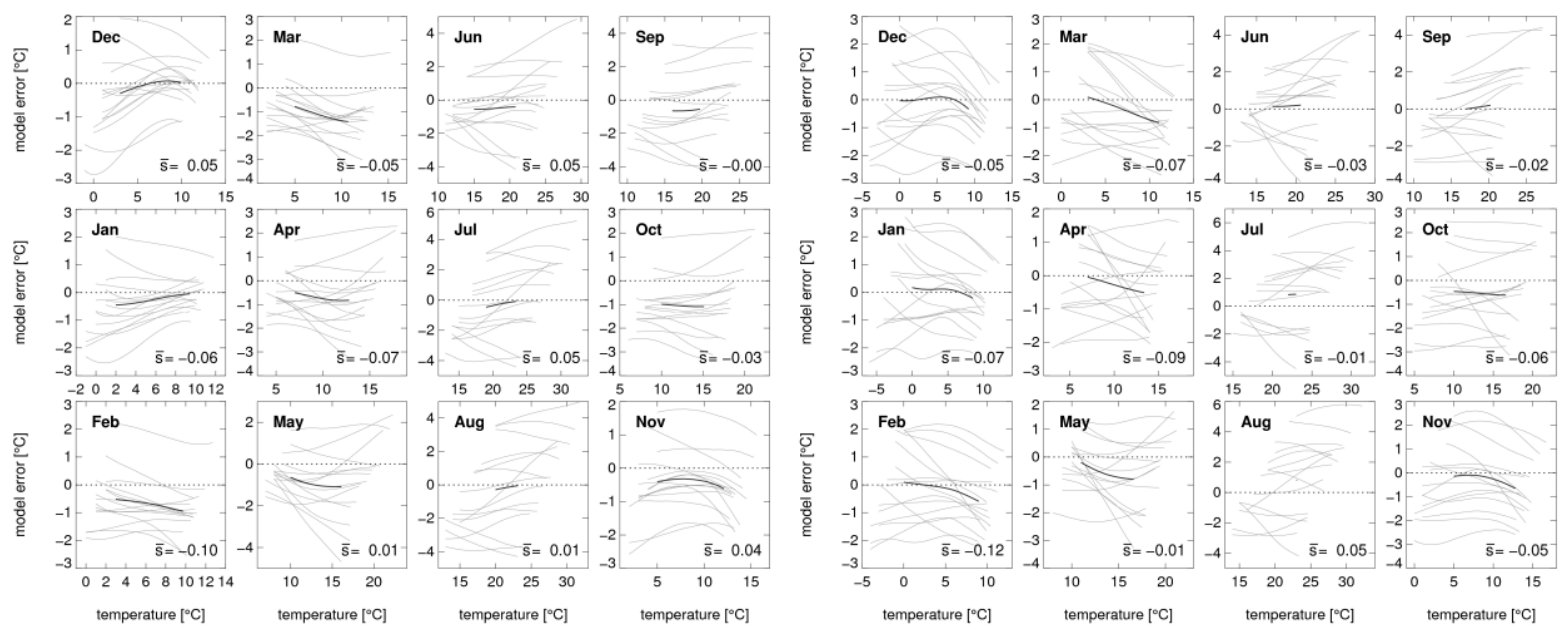

2 Figure S9: Temperature error characteristics (model minus observed) of the ENSEMBLES

3 models in IP (left panels) and MD (right panels). The light lines show the error characteristics

4 of the individual models, the bold line shows the ensemble average. The number in the lower

5 right corner of each panel denotes multi-model average error slope.
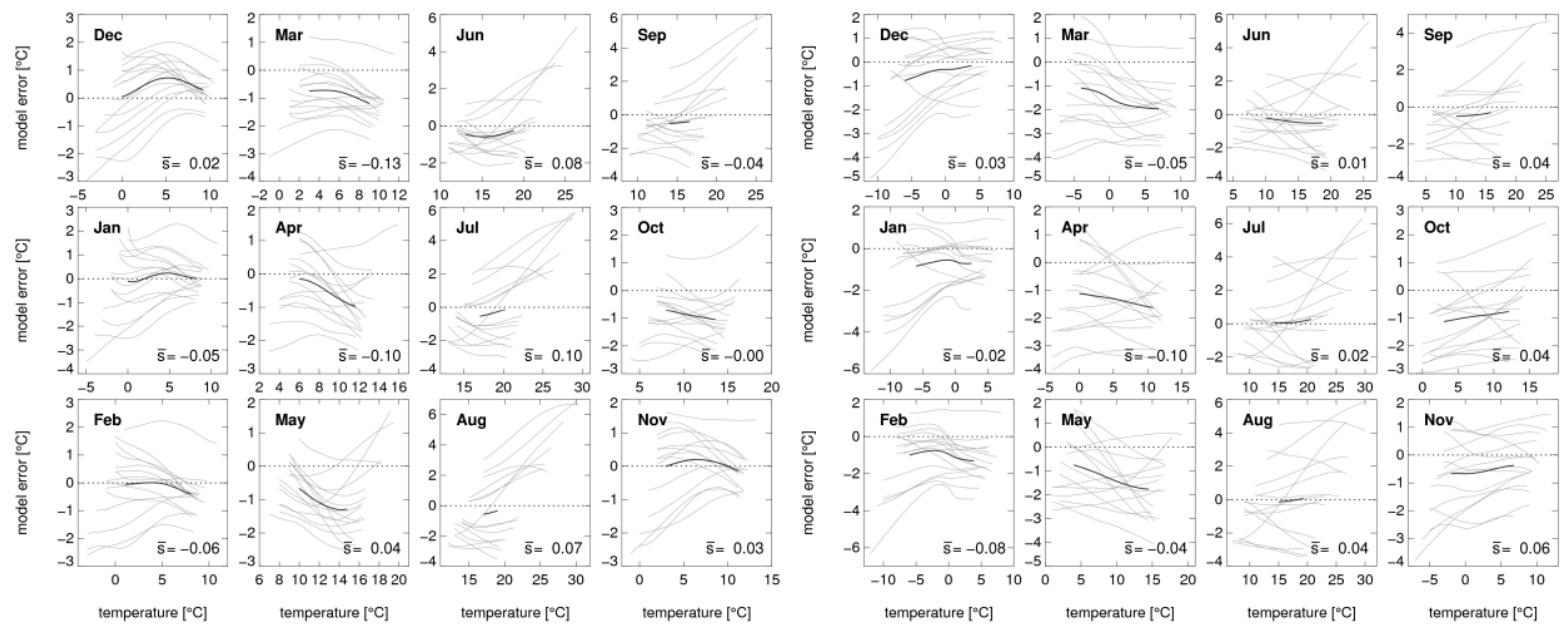

7 Figure S10: Temperature error characteristics (model minus observed) of the ENSEMBLES

8 models over FR (left panels) and AL (right panels). The light lines show the error

9 characteristics of the individual models, the bold line shows the ensemble average. The number in the lower right corner of each panel denotes multi-model average error slope. 

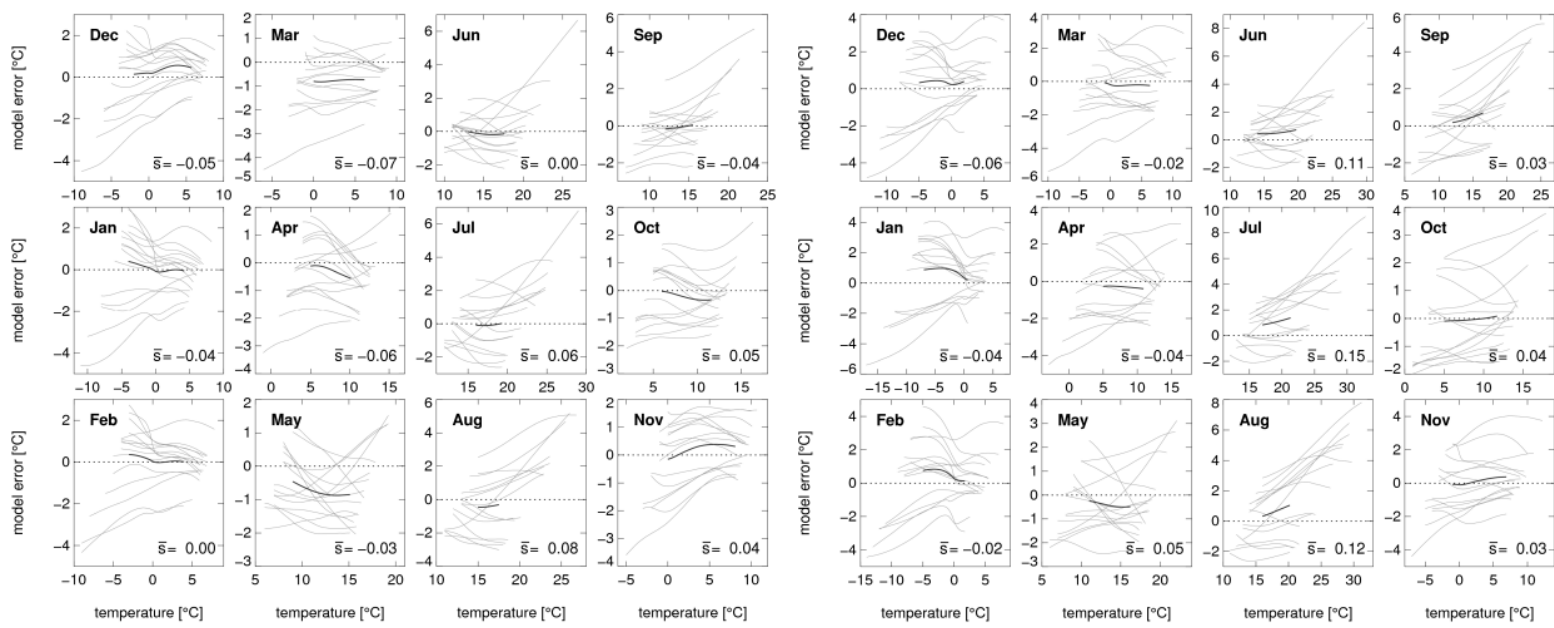

2 Figure S11: Temperature error characteristics (model minus observed) of the ENSEMBLES

3 models over ME (left panels) and EA (right panels). The light lines show the error

4 characteristics of the individual models, the bold line shows the ensemble average. The

5 number in the lower right corner of each panel denotes multi-model average error slope.
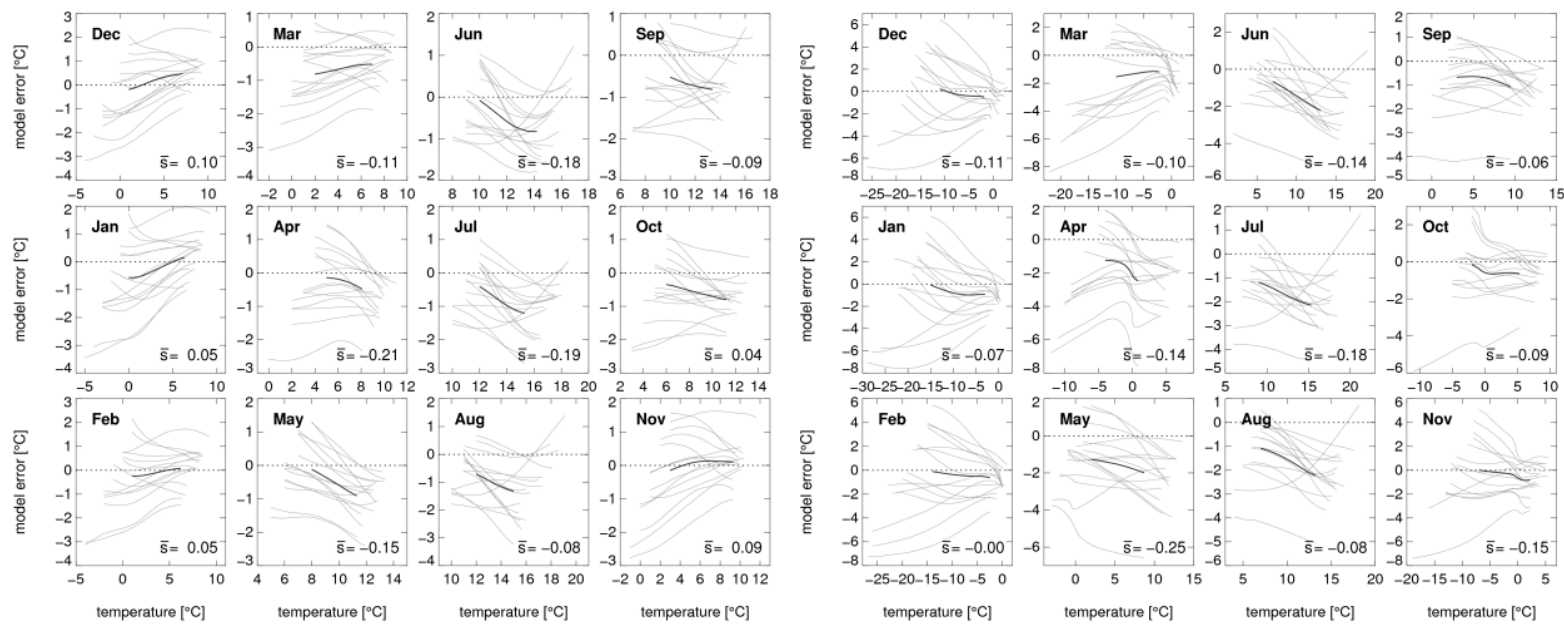

7 Figure S12: Temperature error characteristics (model minus observed) of the ENSEMBLES

8 models over BI (left panels) and SC (right panels). The light lines show the error

9 characteristics of the individual models, the bold line shows the ensemble average. The number in the lower right corner of each panel denotes multi-model average error slope. 


\section{Derivation of Equation (9)}

3 Eq. (8) describes the variance of CCSs in an multi-model ensemble considering intensity-

4 dependent model errors in a linearized way (see Sect. 4 of the original paper). Here we give

5 some details on the derivation of Eq. (9) from Eq. (8).

$6 \quad \operatorname{var}\left(\Delta y^{i}\right)=\frac{1}{n} \sum_{i=1}^{n}\left[\Delta y^{i}-\overline{\Delta y^{i}}\right]^{2}=\frac{1}{n} \sum_{i=1}^{n}\left[\bar{s}\left(\Delta y^{i}-\overline{\Delta y^{i}}\right)+s^{\prime i} \Delta y^{i}+\Delta y_{v}^{i}-\operatorname{cov}\left(s^{\prime i}, \Delta y^{i}\right)\right]^{2}$.

7 Eq. (8) in an expanded form writes:

8

$$
\begin{aligned}
& \operatorname{var}\left(\Delta y^{i}\right)=\frac{1}{n} \sum_{i=1}^{n}\left(\bar{s}^{2}\left(\Delta y^{i}-\overline{\Delta y^{i}}\right)^{2}\right)+\frac{1}{n} \sum_{1}^{n}\left(s^{\prime i^{2}} \Delta y^{i^{2}}\right)+\frac{1}{n} \sum_{i=1}^{n} \Delta y_{v}^{i^{2}}+\operatorname{cov}^{2}\left(s^{\prime i}, \Delta y^{i}\right)+ \\
& \frac{2}{n} \sum_{i=1}^{n}\left(\bar{s}\left(\Delta y^{i}-\overline{\Delta y^{i}}\right) s^{\prime i} \Delta y^{i}\right)-\frac{2}{n} \sum_{i=1}^{n}\left(s^{\prime i} \Delta y^{i} \operatorname{cov}\left(s^{\prime i}, \Delta y^{i}\right)\right)-\frac{2}{n} \sum_{i=1}^{n}\left(\operatorname{cov}\left(s^{i}, \Delta y^{i}\right) \bar{s}\left(\Delta y^{i}-\overline{\Delta y^{i}}\right)\right)
\end{aligned}
$$

9 The term $\frac{1}{n} \sum_{i=1}^{n} \Delta y_{v}^{i^{2}}$ equals $\operatorname{var}\left(\Delta y_{\nu}{ }_{\nu}\right)$ and $\frac{1}{n} \sum_{i=1}^{n} \bar{s}^{2}\left(\Delta y^{i}-\overline{\Delta y^{i}}\right)^{2}$ equals $\bar{s}^{2} \operatorname{var}\left(\Delta y^{i}\right)$. This

10 results in:

11

$$
\operatorname{var}\left(\Delta y^{i}\right)=\bar{s}^{2} \operatorname{var}\left(\Delta y^{i}\right)+\frac{1}{n} \sum_{1}^{n}\left(s^{\prime i^{2}} \Delta y^{i^{2}}\right)+\operatorname{var}\left(\Delta y^{i}{ }_{v}\right)+\operatorname{cov}^{2}\left(s^{\prime i}, \Delta y^{i}\right)+
$$

$$
\frac{2}{n} \sum_{i=1}^{n}\left(\bar{s}\left(\Delta y^{i}-\overline{\Delta y^{i}}\right) s^{\prime i} \Delta y^{i}\right)-\frac{2}{n} \sum_{i=1}^{n}\left(s^{i} \Delta y^{i} \operatorname{cov}\left(s^{\prime i}, \Delta y^{i}\right)\right)-\frac{2}{n} \sum_{i=1}^{n}\left(\operatorname{cov}\left(s^{i}, \Delta y^{i}\right) \bar{s}\left(\Delta y^{i}-\overline{\Delta y^{i}}\right)\right)
$$

12 The $5^{\text {th }}$ term on the right hand side can be written as:

$13 \quad \frac{2}{n} \sum_{i=1}^{n}\left(\bar{s}\left(\Delta y^{i}-\overline{\Delta y^{i}}\right) s^{i^{i}} \Delta y^{i}\right)=\frac{2 \bar{s}}{n} \sum_{i=1}^{n}\left(\Delta y^{i} s^{\prime i} \Delta y^{i}\right)-\frac{2 \bar{s} \overline{\Delta y^{i}}}{n} \sum_{i=1}^{n}\left(s^{\prime i} \Delta y^{i}\right)$.

14 Using the definition of covariance $(\operatorname{cov}(x, y)=E(x y)-E(x) E(y)$, E being the expectation

15 value), the $2^{\text {nd }}$ term on the right hand side of Eq. (S2) can be rewritten as:

$16 \quad \frac{2 \bar{s}}{n^{2}} \sum_{i=1}^{n} \Delta y^{i} \sum_{i=1}^{n} s^{i} \Delta y^{i}+2 \bar{s} \operatorname{cov}\left(\Delta y^{i}, s^{i} \Delta y^{i}\right)$

17 and further expanded to:

$18 \quad 2 \bar{s}{\overline{\Delta y^{i}}}^{2} \frac{1}{n} \sum_{i=1}^{n} s^{i}+2 \bar{s} \overline{\Delta y^{i}} \operatorname{cov}\left(s^{i}, \Delta y^{i}\right)+2 \bar{s} \operatorname{cov}\left(\Delta y^{i}, s^{i} \Delta y^{i}\right)$. 
1 Here, the first term is zero due to the zero expectation of $s^{i}$. Eq. (S3) now reads:

$2 \quad \frac{2}{n} \sum_{i=1}^{n}\left(\bar{s}\left(\Delta y^{i}-\overline{\Delta y^{i}}\right) s^{i} \Delta y^{i}\right)=2 \bar{s} \operatorname{cov}\left(\Delta y^{i}, s^{i} \Delta y^{i}\right)$.

3 Eq. (S2) can now be written as:

$$
\operatorname{var}\left(\Delta y^{i}\right)=\bar{s}^{2} \operatorname{var}\left(\Delta y^{i}\right)+\frac{1}{n} \sum_{1}^{n}\left({s^{\prime}}^{2} \Delta y^{i^{2}}\right)+\operatorname{var}\left(\Delta y^{i}{ }_{v}\right)+\operatorname{cov}^{2}\left(s^{\prime i}, \Delta y^{i}\right)+
$$

$$
2 \bar{s} \operatorname{cov}\left(\Delta y^{i}, s^{\prime i} \Delta y^{i}\right)-\frac{2}{n} \sum_{i=1}^{n}\left(s^{\prime i} \Delta y^{i} \operatorname{cov}\left(s^{\prime i}, \Delta y^{i}\right)\right)-\frac{2}{n} \sum_{i=1}^{n}\left(\operatorname{cov}\left(s^{\prime i}, \Delta y^{i}\right) \bar{s}\left(\Delta y^{i}-\overline{\Delta y^{i}}\right)\right)
$$

5 The term $-\frac{2}{n} \sum_{i=1}^{n}\left(s^{i} \Delta y^{i} \operatorname{cov}\left(s^{i}, \Delta y^{i}\right)\right)$ is equal to $-2 \operatorname{cov}^{2}\left(s^{\prime i}, \Delta y^{i}\right)$ due to similar reasoning as

6 used above. In addition, the term $-\frac{2}{n} \sum_{i=1}^{n}\left(\operatorname{cov}\left(s^{\prime i}, \Delta y^{i}\right) \bar{s}\left(\Delta y^{i}-\overline{\Delta y^{i}}\right)\right)$ disappears, since the

7 expectation of $\left(\Delta y^{i}-\overline{\Delta y^{i}}\right)$ is zero by definition. Eq. (S5) can now be simplified to:

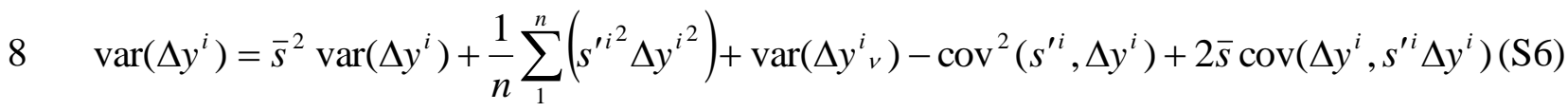

9 Finally, the term $\frac{1}{n} \sum_{1}^{n}\left(s^{i^{2}} \Delta y^{i^{2}}\right)$ can be written as

$10 \quad \frac{1}{n} \sum_{1}^{n}\left(s^{\prime i} \Delta y^{i}\right) \frac{1}{n} \sum_{1}^{n}\left(s^{\prime i} \Delta y^{i}\right)+\operatorname{cov}\left(s^{\prime i} \Delta y^{i}, s^{\prime i} \Delta y^{i}\right)$

11 Using the similarity $\operatorname{cov}(x, x)=\operatorname{var}(x)$ and similar reasoning as before, this expression can be

12 written as $\operatorname{cov}^{2}\left(s^{i}, \Delta y^{i}\right)+\operatorname{var}\left(s^{\prime i} \Delta y^{i}\right)$, which enables to further simplify Eq. (S5) to its final

13 form:

14

$$
\operatorname{var}\left(\Delta y^{i}\right)=\operatorname{var}\left(\Delta y^{i}{ }_{v}\right)+\bar{s}^{2} \operatorname{var}\left(\Delta y^{i}\right)+\operatorname{var}\left(s^{i} \Delta y^{i}\right)+2 \bar{s} \operatorname{cov}\left(\Delta y^{i}, s^{i} \Delta y^{i}\right) .
$$

